\title{
The mysterious cut-off of the Planetary Nebula Luminosity Function
}

\author{
K. Gesicki*, A. A. Zijlstra, and M. M. Miller Bertolami
}

\section{Letter to Nature Astronomy}

Planetary Nebulae (PNe) mark the end of the active life of $90 \%$ of all stars. They trace the transition from a red giant to a degenerate white dwarf. Stellar models $\frac{1,2}{2}$ predicted that only stars above approximately twice the solar mass could form a bright PN. But the ubiquitous presence of bright $\mathrm{PNe}$ in old stellar populations, such as elliptical galaxies, contradicts this: such high mass stars are not present in old systems. The planetary nebula luminosity function (PNLF), and especially its bright cut-off, is almost invariant between young spiral galaxies, with high mass stars, and old elliptical galaxies, with only low mass stars. Here we show that new evolutionary tracks of low-mass stars are capable to explain in a simple manner the decades-old mystery. The agreement between the observed PNLF and stellar evolution validates the latest theoretical modelling. With these models, the PNLF provides a powerful diagnostic to derive star formation histories of intermediate-age stars. The new models predict that the Sun at the end of its life will also form a PN, but it will be faint.

Low and intermediate mass stars, up to about $8 M_{\odot}$, end their lives with a phase of extreme mass loss. The superwind ejects the envelope, leaving only the degenerate core behind. The core briefly ionizes the ejecta, before entering the terminal white dwarf cooling stage. The ionized ejecta remain visible as a planetary nebula (PN) for thousands of years, before dispersing into the interstellar medium $\stackrel{3}{ }$.

PNe form the most luminous phase of evolution of their host stars, with typical luminosity $L \sim 10^{4} L_{\odot}$. In addition, much of the nebular luminosity comes out in a few bright, narrow emission lines. The brightest line, [O III] $5007 \AA$, can emit as much as $10^{3} L_{\odot}$. This makes PNe detectable to very large distances, including galaxies beyond the Virgo cluster. The PNLF has been established as an important extragalactic distance estimator $\underline{4.5}^{-5}$. However, stellar evolution models have thus far been unable to explain it $\underline{\underline{6}}$.

The PNLF describes the fraction of PNe in a galaxy at each specified luminosity bin ${ }^{7}$. For the emission line [O III] $5007 \AA$, the absolute magnitude is 


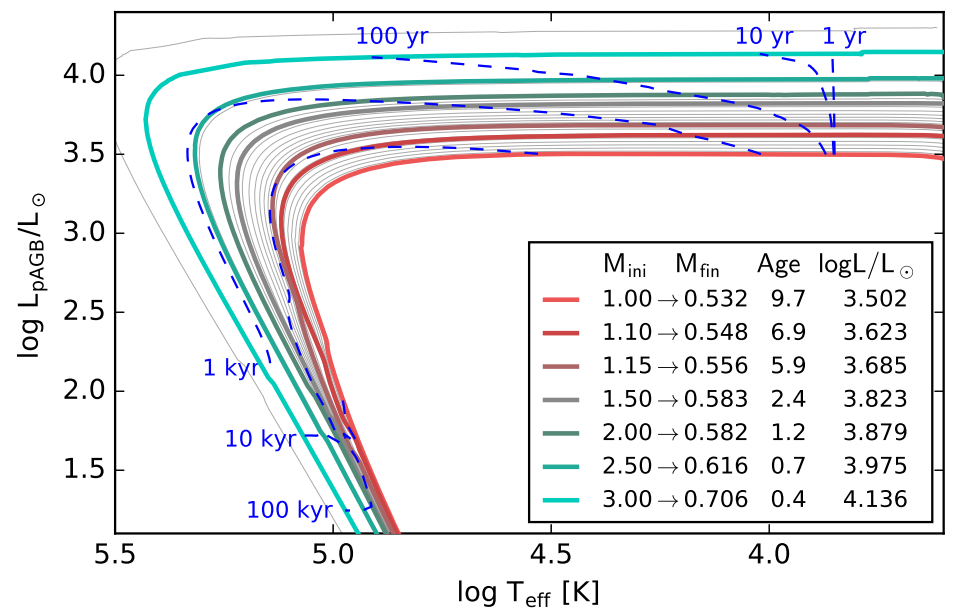

Figure 1: Stellar evolution sequences and timescales. The coloured lines present the post-AGB stellar evolution sequences $\frac{14}{14}$ adopted for the computations of the fluxes shown in Fig.2. Blue dashed lines indicate isochrones at selected post-AGB ages. Thin grey lines show the interpolated sequences used for the construction of the PNLFs shown in Fig. 3. The inset presents the model parameters for the seven computed tracks: the initial and final masses in $M_{\odot}$ units, the total age since ZAMS in Gyr and the $\log L / L_{\odot}$.

defined $\stackrel{8}{ }$ as $M_{5007}=-2.5 \log (F[\mathrm{O} I I I])-13.74$. Here $F([\mathrm{O} I I I])$ is the line flux in units of $\mathrm{erg} \mathrm{s}^{-1} \mathrm{~cm}^{-2}$, assuming a distance of $10 \mathrm{pc}$. In these units, the PNLF shows a well-defined, steep cut-off at the bright end, at $M_{5007}^{*} \simeq-4.5$. The observed value of $M_{5007}^{*}$ is almost invariant between different galaxies, with a scatter of only $\sim 0.17 \mathrm{mag} \frac{5}{5}$ for metal-rich galaxies. The observed magnitudes include the effect of extinction within the circumstellar material of the PN, which especially for very young and compact $\mathrm{PNe}$ can be of the order $\sim 0.5$ mag.

To reproduce the invariant cut-off some studies $\frac{9}{}$ assumed a final stellar mass distribution with an upper cut-off at $0.63 M_{\odot}$ (initial mass: $\left.M_{\mathrm{i}} \approx 2 M_{\odot}\right)$. This is much too high for older stellar populations $\underline{10}$. Other studies $11, \underline{12}$ showed that the stellar evolution models available before $2016 \stackrel{1,2}{\underline{1}}$, combined with nebular models of evolving optical thickness, predicted a brightening of $M_{5007}^{*}$ by more than 4 magnitudes when going from old (10 Gyr) to young (1 Gyr) stellar populations, in stark contradiction with the observed invariance of $M_{5007}^{*}$. These inconsistencies are a long standing mystery in the study of the PNLF, and have thrown doubts on our understanding of the final evolution of low mass stars, including the Sun.

Recently, the details of the stellar evolution models 1.2 applied in the modelling of the PNLF have been questioned. The analysis of PNe in the Galactic bulge $\frac{13}{}$ suggested that the speed of the stellar evolution during the PN phase 
had been underestimated by a factor of three or more. Independently, new stellar evolution models for post-asymptotic giant branch (post-AGB) stars were developed $\underline{14}$, with a carefully computed post-AGB phase based on new opacities, an updated treatment of the stellar physics and a proper account of previous evolutionary stages (see Fig.11). These new models have very different initialfinal mass relations calibrated to be in a good agreement with observations. Their post-AGB evolution is faster and brighter than the previous models. The new models fundamentally change the interpretation of the PNLF.

From this new model grid, we selected seven evolutionary sequences with metallicity $Z=0.01$, representative for a wide range of Solar-like populations. The initial masses range from 1 to $3 M_{\odot}$ and the final masses from 0.532 to $0.706 M_{\odot}$ respectively. This initial mass range corresponds to stellar ages between 0.5 and 10 Gyr (see the inset in Fig.11). The grid includes previously published sequences $\frac{14}{}$ and newly computed models using the same physical assumptions.

The evolutionary sequence provides the temperature evolution and the luminosity evolution of the post-AGB star. To model the PNLF we calculate emission line fluxes of model nebulae surrounding each one of the stellar models. The complete photoionization structures of the nebulae were calculated using the Torun codes $\frac{15}{}$, to derive the flux of the single nebular line used, [O III] $5007 \AA$.

We first ran models with a non-evolving constant density shell, with a fixed inner radius of $0.01 \mathrm{pc}$, outer radius of $0.02 \mathrm{pc}$, and a mass of $0.15 M_{\odot}$. These parameters were chosen to result in ionization bounded (opaque) nebulae and were adopted for each of the seven evolutionary sequences. In this maximumnebula hypothesis, the fluxes emitted at each phase of the stellar evolution are maximized. It is appropriate for testing whether these PNe can reproduce the peak of the PNLF.

For each evolutionary sequence we computed the fluxes emitted in the line [O III] $5007 \AA$, converted to the magnitude scale $M_{5007}$. Fig.2 2 shows this magnitude evolution during the post-AGB evolution for the seven low-mass stars introduced in Fig. 11 The most massive track reaches magnitudes above the observed PNLF cut-off value. However the time spent at maximum brightness is negligible in comparison with the average life-time of PNe: the typical observed kinematic age of PNe is between $\lesssim 1000$ and $10,000 \mathrm{yrs}$. In addition, the highmass, youngest PNe are expected to be more compact and may have significant circumstellar extinction. The next five less-massive tracks behave very regularly and over thousands of years they show an [O III] brightness very close to the observed cut-off value, exceeding it (if at all) by only a fraction of a magnitude. This is a consequence of the very similar post-AGB luminosity of these models (see the inset in Fig. (1) which changes by only 0.25 dex between the $1.1 M_{\odot}$ and the $2 M_{\odot}$ sequences (ages $7 \mathrm{Gyr}$ and $1 \mathrm{Gyr}$ respectively). Finally, the least massive track, at $1 M_{\odot}$ evolves so slowly that the ejected nebula will be dispersed before the star culminates in $T_{\text {eff. }}$. The new stellar evolution models coupled with photoionization modelling under the maximum-nebula hypothesis predict the maximum nebular [O III] brightness to be close to the observed value over 


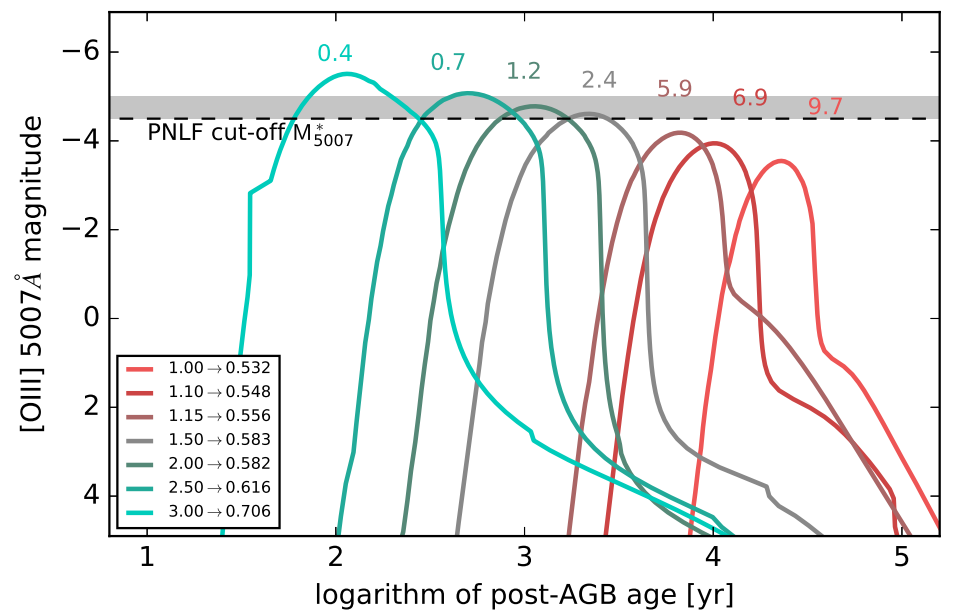

Figure 2: Evolution of [O III] $5007 \AA$ fluxes against logarithmic time for the maximum-nebula hypothesis. The solid lines represent evolutionary tracks of central stars, with initial and final masses as given in the inset. The numbers above each curve indicate the total age in Gyr of a given stellar model. The PNLF canonical cut-off value of $M_{5007}=-4.5 \mathrm{mag}$ is shown as a horizontal dashed line; the grey band represents the possible range of this value when correction for extinction was considered.

a large range of stellar ages and masses (Fig.22). This already suggests that the models can reproduce the PNLF cut-off for a variety of stellar populations.

Next, we allow the modelled gaseous shell to evolve together with the central star. We assumed again a constant density shell with fixed inner radius of $0.01 \mathrm{pc}$ and fixed total mass of $0.10 M_{\odot}$, but now the outer radius expands from $0.02 \mathrm{pc}$ up to $0.5 \mathrm{pc}$ at a constant velocity. The expansion of the shell results in a transition of the nebula from opaque to transparent to the ionizing radiation. Varying the kinematics provides different [O III] brightness scenarios. In the following we will discuss two such scenarios: one where the PN is predominantly opaque during the stellar evolution (the intermediate-nebula hypothesis) and the other one where the $\mathrm{PN}$ is predominantly transparent, the minimum-nebula hypothesis.

To derive the PNLF, we integrate over the stellar mass distribution, assuming a Salpeter initial mass function (IMF) with $n(M) \propto d M \times M^{-2.35}$. We interpolate between the stellar models (Fig. (1) to get a grid of tracks at equally spaced ages. Fig. 3 combines the models to predict PNLFs for both the intermediate and minimum-nebulae hypothesis, and for four different star formation histories (SFHs), appropriate to different galaxy types. The filled histograms (dark for the intermediate-nebula hypothesis with mostly opaque PNe, pale for the minimum-nebula hypothesis with more transparent PNe) use a constant 

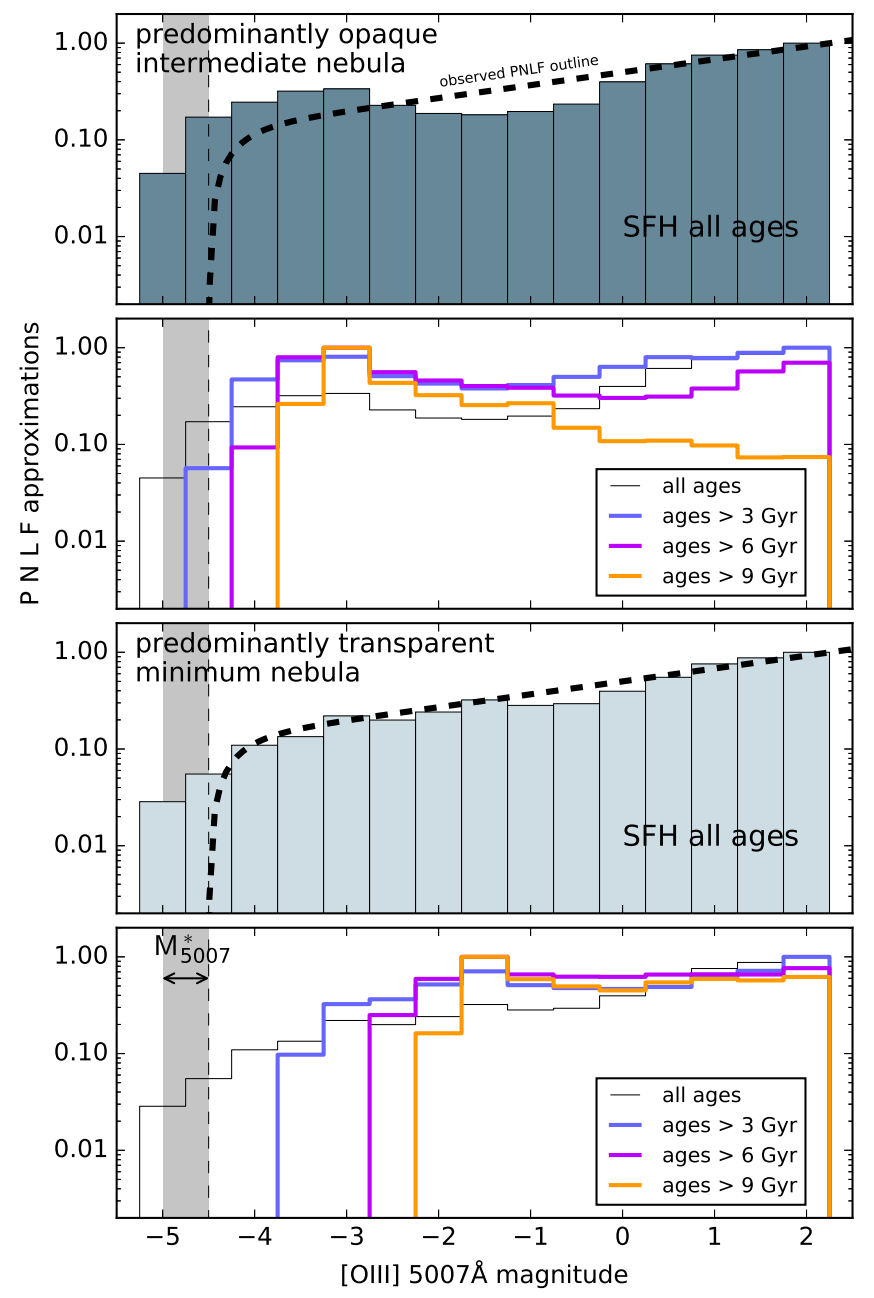

Figure 3: The synthesized PNLF for the intermediate-nebula and minimum-nebula hypothesis, for different star formation histories. We interpolated between evolutionary tracks (Fig.11) to obtain a grid of models sampled at uniform age. For each assumed SFH we combined the individual PNLFs from each gridded model, with weighting factors determined by the SFH, the mass difference between adjacent interpolated tracks, and assuming a Salpeter initial mass function. The PNLF synthesis was performed for two versions of a very simple nebular evolution scenarios. The upper two panels presents the PNe that stay opaque for most of the post-AGB evolution (expansion of the shell starts later and with lower velocity), the bottom two panels concern PNe that become transparent relatively early (expansion starts earlier and with higher velocity). The shaded histograms show the PNLF obtained with a continuous SFH since some $10 \mathrm{Gyr}$ ago until now. The coloured lines show simulations with star formation ending 3, 6, and 9 Gyr ago. Both shaded histograms are supplemented with the observed PNLF outline that combines $\underline{20}$ the bright steep end and the faint tail. At the left side of all panels the canonical value of $M_{5007}^{*}$ is indicated as a vertical dashed line with a grey band representing the possible range of this value when correction for extinction was considered. 
SFH, approximating a non-interacting spiral galaxy. The other two diagrams (shown with coloured lines) use a truncated SFH where a constant star formation ceased 3, 6 and 9 Gyr ago, typical for an elliptical galaxy and for an old stellar population, formed in a burst about 9 Gyr ago.

For the intermediate-nebula hypothesis (the two upper panels in Fig. 3 ), the steep bright-end cut-off of the PNLF appears in our simulations for both the continuous and truncated star formation histories, at a value very close to the canonical observed limit of $M_{5007}^{*} \sim-4.5$. The brightest PNe are created by progenitors $1.1 M_{\odot}<M_{\mathrm{i}}<2 M_{\odot}$, which have nearly the same post-AGB stellar luminosity, $\log \left(L_{\mathrm{pAGB}} / L_{\odot}\right)=3.75 \pm 0.13$, and have total ages from 1 to $\sim 7 \mathrm{Gyr}$. The initial stellar masses are significantly smaller than was required in similar older models. The fact that the cut-off appears at the right magnitude over a wide age range of stellar populations provides a simple solution to the long standing mystery of the observed PNLF cut-off invariance.

The two lower panels in Fig. 3 present the minimum-nebula hypothesis. It reproduces the observed faint-tail behaviour of PNLF, however the bright-end becomes progressively fainter with age of the population.

We conclude that the PNLF can be reproduced using the new stellar models, under the assumptions that the ejected shells can be approximated with the intermediate-nebula hypothesis, and that the originating stellar population harbours a significant number of stars with ages of 3-7 Gyr. The age range is consistent with the known SFH, where nearby galaxies with masses between $10^{10}$ and $10^{11} M_{\odot}$ tend to have declining star formation rates since $3-5$ Gyr ago 16 . Elliptical galaxies also can contain a fraction of stars in this age range 17 . Our results confirm previous studies ${ }^{18}$ which concluded that appropriate PNLF peak luminosities could be attained by lower-mass stars if the nebulae remained optically thick during most of their evolution.

The necessary condition that the modelled spherical PNe remain opaque during a significant part of their early evolution can perhaps be satisfied by a high opacity in one direction and transparency in another. For bipolar PNe containing a dense equatorial torus and thin lobes, 3D modelling $\frac{19}{}$ reproduces well the line emissivities (including [O III]). The details of the evolution and expansion of PNe are still not well understood. Our results puts constraints on this evolution, for those PNe which make up the peak of the PNLF.

The brightness and the evolutionary speed of the central stars of PNe, both crucial to the PNLF, are influenced by physical processes on the previous AGB phase, in particular the mass loss and the mixing which bring the products of nuclear burning to the surface. The details of the observed PNLF may put constraints on the efficiency of these still poorly understood processes in AGB stars.

The models give new information about the final fate of the Sun. In our models, it is near the low-mass limit for PN formation. The Sun still reaches a temperature high enough to ionize the ejecta, before they disperse. Hydrogen ionization begins 5000 years after the end of the AGB, and [O III] appears from 12,500 years. The stellar luminosity suffices to put its PN within a magnitude of the bright cut-off of the PNLF. However, at this late time, it is expected that 
the ejecta will no longer be optically thick, and therefore the PN may be rather fainter than this. If the Sun leaves the AGB during the helium burning phase of the thermal pulse cycle, its luminosity and speed of evolution will be three times lower and no PN will form. The Sun is close to the lowest mass star that can still produce a $\mathrm{PN}$.

\section{Methods}

\section{Extinction corrections to the PNLF cut-off}

The extinction towards extragalactic PNe is a combination of foreground extinction (within our Galaxy), local extinction (within the host galaxy) and circumstellar extinction (within the stellar ejecta). The foreground is normally constant across the host galaxy and is known. The local extinction is a stochastic parameter, which mostly affects PNe located within the disk of spiral galaxies. Circumstellar extinction stems from dust located in the non-ionized regions surrounding the PN. Observed PNLF are generally corrected for foreground extinction, but not for the poorly known local and circumstellar extinction.

We calculate the circumstellar extinction using the equation 27

$$
A_{\mathrm{V}} \approx 0.5\left[\frac{\dot{M}}{10^{-5} \mathrm{M}_{\odot} \mathrm{yr}^{-1}}\right]\left[\frac{v_{\exp }}{15 \mathrm{~km} \mathrm{~s}^{-1}}\right]^{-1}\left[\frac{r}{10^{16} \mathrm{~cm}}\right]^{-1} .
$$

This assumes a standard ratio of $N_{\mathrm{H}_{2}}=2 \times 10^{21} A_{\mathrm{V}} \mathrm{cm}^{-2} \mathrm{mag}^{-1}$, appropriate for interstellar silicate dust $\underline{28}$. For our minimum radius of $0.01 \mathrm{pc}$ and assuming $\dot{M}=5 \times 10^{-5} M_{\odot} \mathrm{yr}^{-1}$ and $v_{\exp }=15 \mathrm{~km} \mathrm{~s}^{-1}$, this predicts $A_{\mathrm{V}} \approx 0.8 \mathrm{mag}$. The smallest radii of Galactic PNe are around $0.03 \mathrm{pc}^{29}$, giving an expected $A_{\mathrm{V}} \approx 0.25 \mathrm{mag}$.

As a test, the well studied PNe population of the outer disk of the Andromeda galaxy M31 is shown in Fig. (4. The foreground extinction to M 31 amounts to $A_{\mathrm{V}}=0.175$. The corresponding value of $A_{5007}$ is presented as the horizontal dotted line. The coloured symbols show $M_{5007}$ corrected for foreground extinction only, and the thin lines indicate the shift when corrected for the total extinction, which here is derived from the spectra. The majority of objects have additional extinctions of a few tenths of a magnitude or less. A few have higher extinction. After correcting for the full extinction, the value of the cut-off of the PNLF shows a small shift from $M_{5007}^{*}=-4.2$ to -4.5 . In our models, the former value would indicate an age of the stellar population of around $7 \mathrm{Gyr}$, while the corrected value corresponds to stellar ages of less than $6 \mathrm{Gyr}$. This illustrates the diagnostic power of the PNLF to constrain star-formation histories, provided accurate extinctions are available. In this age range, few other diagnostics are available. It should however be noted that extinction corrections can be uncertain, especially if there is significant circumstellar extinction and scattering, potentially involving non-standard dust grains ${ }^{30}$. 


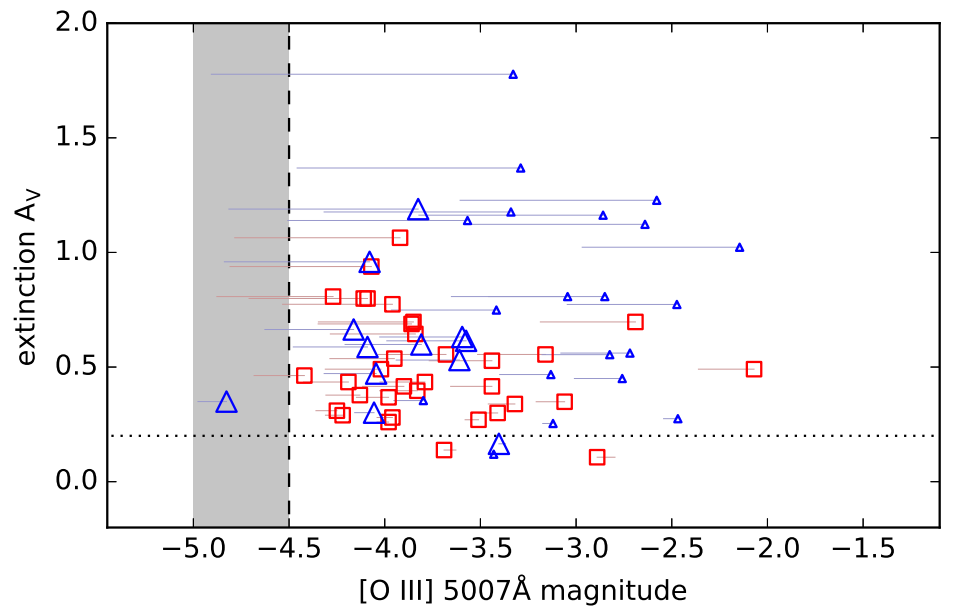

Figure 4: Observed values of $M_{5007}$ for planetary nebulae of the outer disk of the Andromeda galaxy, M 31. The data is taken from different sources, shown as red squares $21,22,23,24$ and blue triangles $\frac{25}{2}$. Extinctions were calculated from the published $\mathrm{H} \alpha / \mathrm{H} \beta$ ratio, assuming a ratio of 2.85. $M_{5007}$ was taken from a single source $\frac{26}{}$, with one exception ${ }^{25}$ where $M_{5007}$ is measured from the spectra itself. The small triangles indicate objects where the extinction is less accurate because of low $\mathrm{S} / \mathrm{N}$ on the $\mathrm{H} \beta$ line. The foreground extinction is $A_{\mathrm{V}}=0.175$, extinction at $[\mathrm{O} \mathrm{III}]$ is taken as $1.2 A_{\mathrm{V}}$, and the distance modulus to M 31 is taken as 24.47. The coloured symbols show $M_{5007}$ corrected for foreground extinction; the thin lines indicate the $M_{5007}$ magnitudes after correction for the total extinction. The canonical value of $M_{5007}^{*}=-4.5$ is shown as the vertical dashed line. The grey vertical band represents the corresponding range of values assuming extinction correction up to $0.5 \mathrm{mag}$. The horizontal dotted line represents the foreground component to $A_{5007}$.

The grey band in the plots shows the dimming effect a circumstellar extinction of up to $0.5 \mathrm{mag}$ would have on the observed PNLF, when compared to the theoretical, extinction-free curves. Such an extinction is possible for the most compact $\mathrm{PNe}$, from the above calculations.

\section{La Plata stellar evolution code}

LPCODE is a one-dimensional stellar evolution code that has been widely used for the computation of full evolutionary sequences from the zero age main sequence to the white dwarf stage $31,32,33$. The last version of LPCODE 14 includes a state-of-the-art treatment of atomic, molecular and conductive opacities $\frac{34,35,36}{1}$ as well as a detailed treatment of stellar winds and convective boundary mixing. Specifically, LPCODE takes into account both for carbon- and 
oxygen-rich compositions for the determination of both opacity and the stellar wind regime. In addition, convective boundary mixing has been carefully calibrated at different evolutionary stages to reproduce several AGB and post-AGB observables $\frac{14}{2}$. The post-AGB evolution of the seven tracks computed with this code and discussed in this article are presented in Fig. 1. Each star evolves first at constant luminosity to higher temperatures, followed by a rapid fading and slow cooling. The speed of evolution is much faster at higher stellar mass, so that lower-mass stars spend much longer at their peak luminosity than higher mass stars do. A metallicity of $Z=0.01$ was selected, as being appropriate for sun-like stars. The post-AGB tracks have little dependence on metallicity, and using $Z=0.02$ would cause only minor changes to the derived masses and ages of the stars. A $1.25 M_{\odot}$ track $\frac{14}{14}$ was not used for the interpolations because it was affected by a thermal pulse unusually close to the AGB turn-off which affected the subsequent evolution. The $1 M_{\odot}$ presented here was adjusted to ensure it left the AGB during the hydrogen burning phase, by briefly turning off the stellar wind until the hydrogen shell had fully activated.

\section{Torun photoionization code}

The Torun codes were written in their original form some twenty years ago $\underline{15}$. The central star is approximated as a black-body. The nebula is approximated with a spherically symmetric shell having assumed inner and outer radii and radial density distribution. The chemical composition of the nebular gas was taken from the data set named PLANetary which is included as a part of the CLOUDY $\operatorname{code} \mathrm{e}^{37}$. No dust presence was assumed. The ionization state of gas was computed by solving the equations of statistical equilibrium and iterated to convergence with the kinetic temperature and electron density. These codes were not written in a form suitable for public availability but they are easy to adapt to different needs and run very fast on standard workstations. They have been used in numerous publications.

\section{[O III] magnitudes for individual stellar tracks}

We first derived [O III] fluxes from a single evolutionary track, for a particular stellar mass. At this step we were interested in maximizing the flux emitted in the [O III] line. The shell parameters were adopted to secure that the PN stays opaque during the whole stellar evolution, the maximum-nebula hypothesis. As the stellar temperature increases and the number of ionizing photons goes up, the ionization front expands and the ionized mass increases. To obtain opaque $\mathrm{PNe}$, the total mass of the shell should exceed the ionized mass. We found that a constant density shell of inner radius of $0.01 \mathrm{pc}$, outer radius of $0.02 \mathrm{pc}$ and total mass of $0.15 M_{\odot}$ fulfils our demands for each of the central star tracks. The resulting hydrogen number density $\sim 1.5 \cdot 10^{5} \mathrm{~cm}^{-3}$ guarantees confinement of the ionization front even for the hottest stars. These parameters remain typical for unevolved PNe. For each of the tracks presented in Fig.1 the flux evolution in the [O III] line was calculated using the Torun model described 
above. The [O III] line flux we converted to magnitude scale and in this way the dependencies shown in Fig.2 were obtained.

We derived also the central star luminosity fraction that is reprocessed into nebular flux in [O III] $5007 \AA$ line. The maxima of this reprocessing efficiency remain nearly constant, at the value $\sim 0.12$ which is in agreement with earlier modelling 38 . However the actual values of this efficiency vary significantly with stellar evolution, firstly they increase with $T_{\text {eff }}$ then start to decrease because of higher oxygen ionization and further decrease because the star enters the cooling track. In fact they follow closely the time dependencies of [O III] $5007 \AA$ fluxes drawn in Fig.2.

\section{PNLF for expanding PNe in composite stellar populations}

To account for simultaneous evolution of the central star and the expansion of nebular shell we applied a simple scheme. We kept the nebular inner radius fixed and the total nebular mass constant. The shell is assumed to have a constant density with radius. We specified the outer radius as expanding with a constant velocity. The expansion of the outer radius together with a constant inner radius agrees with the observed velocity fields, which have the highest expansion velocities at the outer edge and very low velocity near the inner radius. The expansion results in a fast decrease of the gas density and in consequence of the nebular opacity. The model with expansion starting at the moment when the star leaves the AGB almost immediately becomes transparent. Hydrodynamic calculations $\underline{3}^{\underline{3}}$ show a brief phase of acceleration, caused by the overpressure of the developing ionized region, followed by a plateau. We therefore introduced a delay of the beginning of the (constant) expansion phase. As previously we want to apply the same shell model to all of the synthetic tracks. Because the evolutionary tracks differ significantly in timescales we apply the temperature criterion - the nebula starts to expand after the central star effective temperature reaches a specified value. It does not mean that the early phase is staticas the star heats up the ionization front moves through the opaque shell increasing gradually the nebular ionized radius and the ionized mass. Two parameters define our simplistic models: the threshold temperature which defines the early opaque phase and the expansion velocity which determines the late transparent phase of nebular evolution. As previously the inner radius equals $0.01 \mathrm{pc}$ while the outer radius initially has the value of $0.02 \mathrm{pc}$ and later expands but not more than to $0.5 \mathrm{pc}$. The total mass to be ionized was assumed $0.1 M_{\odot}$, it is a little lower than at previous computations since now we are interested in nebulae that at some moment should become transparent. The resulting hydrogen number densities decrease with post-AGB time from $\sim 10^{5}$ to $\sim 5 \mathrm{~cm}^{-3}$. All these parameters agree with the rather wide limits of physical characteristics that define a $\mathrm{PN} \underline{39}$.

With trial and error we pinpointed two nebular models that represent two representative scenarios. One model is predominantly opaque for all evolutionary tracks: it starts expanding with velocity of $20 \mathrm{~km} \mathrm{~s}^{-1}$ after the star heats-up to $60,000 \mathrm{~K}$. The second model expands a little faster $\left(30 \mathrm{~km} \mathrm{~s}^{-1}\right)$ and expansion 
starts somewhat earlier at the temperature of $40,000 \mathrm{~K}$, this model is predominantly transparent for all simulated tracks. These expansion velocities are typical to PNe. The two models are respectively the intermediate-nebula and the minimum-nebula hypothesis.

The most massive stellar model $\left(M_{\mathrm{i}}=3 M_{\odot}\right)$ in both hypotheses evolves so fast that the nebula stays opaque throughout the complete brightest phase and becomes transparent only very late on the cooling track. Also for the least-massive track $\left(M_{\mathrm{i}}=1 M_{\odot}\right)$ the nebula stays opaque but for the opposite reason - the temperature evolution is very slow. For the five intermediate evolutionary tracks the "opaque period" varies from 600 to 6000 years for the minimum-nebulae hypothesis, while for the intermediate-nebula hypothesis it remains opaque about $50 \%$ longer. In both cases, the periods for the nebulae for staying opaque are within reasonable limits.

The carefully computed set of seven tracks (see Fig. 1) served as the base for interpolation on a dense time-grid. In order to interpolate among the tracks we identified key points corresponding to well-defined post-AGB evolutionary phases. Then, the individual evolutionary tracks have been reduced to the same number of "equivalent points" between these key points. For a given age we then compute the expected mass of the post-AGB remnant $\left(M_{\mathrm{f}}^{\text {synthetic }}\right)$ and construct the interpolated track by interpolating in final mass at equivalent points between the two neighbouring tracks - with masses $M_{\mathrm{f}}^{\text {evol.,A }}$ and $M_{\mathrm{f}}^{\text {evol.,B }}$ such that $M_{\mathrm{f}}^{\text {evol.,A }}<M_{\mathrm{f}}^{\text {synthetic }}<M_{\mathrm{f}}^{\text {evol.,B }}$. We computed synthetic tracks interpolated to a uniform grid in the age of the stellar population, to every $0.5 \mathrm{Gyr}$ in the range from 0.25 Gyr to 9.75 Gyr. This grid forms the base for assembling simple stellar populations.

To build a histogram we divided the magnitude axis into $0.5 \mathrm{mag}$ bins (a value comparable with published observed data). The relative time spent by the evolutionary track within given magnitude limits provides a measure of the probability of finding a $\mathrm{PN}$ in this bin. We interpolated the evolutionary track to a dense time grid and counted the number of such grid-points within each magnitude bin. A step of $5 \mathrm{yr}$ appears sufficient to obtain an adequate PNLF. The post-AGB evolution slows down significantly with decreasing stellar luminosity leading to more numerous faint objects. After some time the PN disperses. We calculate the time spent in each magnitude bin up to the adopted by us 20,000 years limit which is well above the ages that contribute to our histograms and is in agreement with recent PN visibility analysis ${ }^{3}$.

Having this data set we can integrate the PNLF contributions from individual synthetic tracks where the SFH and IMF are used to calculate weighting factors for each track. We simulate a simple, constant SFH started and finished at the given ages. However the equal steps in stellar age translate to steps $d M$ in initial mass $M$ that are increasing with $M$. To account for the IMF we therefore multiplied each individual contribution by the mass step $d M$ and by the Salpeter exponent $M^{-2.35}$ and then summed them up. In this way the histograms in Fig. 3 were obtained separately for opaque and for transparent shells. 


\section{References}

[1] Vassiliadis, E., and Wood, P. R. Post-asymptotic giant branch evolution of low- to intermediate-mass stars. ApJS 92, 125-144 (1994).

[2] Bloecker, T. Stellar evolution of low- and intermediate-mass stars. II. PostAGB evolution. A $A A$ 299, 755 (1995).

[3] Jacob, R., Schönberner, D. and Steffen, M. The evolution of planetary nebulae. VIII. True expansion rates and visibility times. $A \& A$ 558, 78 (2013)

[4] Jacoby, G. H., Ciardullo, R., and Ford, H. C. Planetary nebulae as distance indicators. Astronomical Society of the Pacific Conference Series, 4, 42-56 (1988).

[5] Ciardullo, R. The Planetary Nebula Luminosity Function at the dawn of Gaia. Ap\&SS 341, 151-161 (2012).

[6] Ciardullo, R. The Planetary Nebula Luminosity Function and its Issues. Proceedings of the IAU 29B, 15-19 (2016).

[7] Jacoby, G. H., The luminosity function for planetary nebulae and the number of planetary nebulae in local group galaxies. ApJS, 42, 1-18 (1980)

[8] Allen, C. W., Astrophysical quantities. London: University of London, Athlone Press, 3rd ed. (1973). Formula on page 197.

[9] Mendez, R. H., and Soffner, T. Improved simulations of the planetary nebula luminosity function. A\&A 321, 898-906 (1997).

[10] Méndez, R. H., Teodorescu, A. M., Schönberner, D., Jacob, R., and Steffen, M. Toward Better Simulations of Planetary Nebulae Luminosity Functions. ApJ 681, 325-332 (2008).

[11] Marigo, P., Girardi, L., Weiss, A., Groenewegen, M. A. T., and Chiosi, C. Evolution of planetary nebulae. II. Population effects on the bright cut-off of the PNLF. A\&A 423, 995-1015 (2004).

[12] Schönberner, D., Jacob, R., Steffen, M., and Sandin, C. The evolution of planetary nebulae. IV. On the physics of the luminosity function. $A \& B A$ 473, 467-484 (2007).

[13] Gesicki, K., Zijlstra, A. A., Hajduk, M., and Szyszka, C. Accelerated postAGB evolution, initial-final mass relations, and the star-formation history of the Galactic bulge. A\&A 566, A48 (2014).

[14] Miller Bertolami, M. M. New models for the evolution of post-asymptotic giant branch stars and central stars of planetary nebulae. A $\mathcal{E} A$ 588, A25 (2016). 
[15] Gesicki, K., Acker, A., and Szczerba, R. Modelling the structure of selected planetary nebulae. A\&SA 309, 907-916 (1996).

[16] Heavens, A., Panter, B., Jimenez, R., and Dunlop, J. The star-formation history of the Universe from the stellar populations of nearby galaxies $\mathrm{Na}$ ture, 428, 625-627 (2004)

[17] McDermid, R. M., Alatalo, K., Blitz, L., et al. The ATLAS3D Project XXX. Star formation histories and stellar population scaling relations of early-type galaxies MNRAS, 448, 3484 (2015)

[18] Richer, M. G., McCall, M. L. and Arimoto, N. Theoretical models of the planetary nebula populations in galaxies: The ISM oxygen abundance when star formation stops. A\&AS 122, 215-233 (1997).

[19] Gesicki, K., Zijlstra, A. A. and Morisset, C. 3D pyCloudy modelling of bipolar planetary nebulae: Evidence for fast fading of the lobes $A \mathscr{E} A \mathbf{5 8 5}$, A69 (2016).

[20] Ciardullo, R., Jacoby, G. H., Ford, H. C. and Neill, J. D., Planetary nebulae as standard candles. II - The calibration in M 31 and its companions. ApJ 339, 53-69 (1989)

[21] Kwitter, K. B., Lehman, E. M. M., Balick, B., \& Henry, R. B. C., Abundances of Planetary Nebulae in the Outer Disk of M31 ApJ, 753, 12 (2012)

[22] Corradi, R. L. M., Kwitter, K. B., Balick, B., Henry, R. B. C., \& Hensley, K., The Chemistry of Planetary Nebulae in the Outer Regions of M31 ApJ, 807, $181(2015)$

[23] Fang, X., García-Benito, R., Guerrero, M. A., et al., Chemical Abundances of Planetary Nebulae in the Substructures of M31 ApJ, 815, 69 (2015)

[24] Balick, B., Kwitter, K. B., Corradi, R. L. M., \& Henry, R. B. C., Metal-rich Planetary Nebulae in the Outer Reaches of M31 ApJ, 774, 3 (2013)

[25] Kniazev, A. Y., Grebel, E. K., Zucker, D. B., et al., A Search for Planetary Nebulae with the Sloan Digital Sky Survey: The Outer Regions of M31 AJ, 147, 16 (2014)

[26] Merrett, H. R., Merrifield, M. R., Douglas, N. G., et al., A deep kinematic survey of planetary nebulae in the Andromeda galaxy using the Planetary Nebula Spectrograph MNRAS, 369, 120 (2006)

[27] Olofsson, H, Circumstellar Envelopes, in: Asymptotic Giant Branch Stars (H.J. Habing, H. Olofsson, eds.) (Springer, Berlin, 2003)

[28] Güver, T., \& Özel, F., The relation between optical extinction and hydrogen column density in the Galaxy MNRAS, 400, 2050 (2009) 
[29] Frew, D. J., Parker, Q. A., \& Bojičić, I. S., The H surface brightnessradius relation: a robust statistical distance indicator for planetary nebulae MNRAS, 455, 1459 (2016)

[30] Gray, M. D., Matsuura, M., \& Zijlstra, A. A. Radiation transfer in the cavity and shell of a planetary nebula MNRAS, 422, 955 (2012)

[31] Miller Bertolami, M. M., and Althaus, L. G. Full evolutionary models for PG 1159 stars. Implications for the helium-rich $\mathrm{O}(\mathrm{He})$ stars. A $\& A \mathbf{4 5 4}$, 845-854 (2006).

[32] Renedo, I., et al. New Cooling Sequences for Old White Dwarfs. ApJ 717, 183-195 (2010).

[33] Althaus L. G., Miller Bertolami, M. M., and Córsico A. H. New evolutionary sequences for extremely low-mass white dwarfs. Homogeneous mass and age determinations and asteroseismic prospects. A $\mathscr{E} A$ 557, A19 (2013).

[34] Iglesias, C. A., and Rogers, F. J. Updated Opal Opacities. ApJ 464, 943 (1996).

[35] Cassisi, S., Potekhin, A. Y., Pietrinferni, A., Catelan, M., and Salaris, M. Updated Electron-Conduction Opacities: The Impact on Low-Mass Stellar Models. ApJ 661, 1094-1104 (2007).

[36] Weiss, A., and Ferguson, J. W. New asymptotic giant branch models for a range of metallicities. AESA 508, 1343-1358 (2009).

[37] Ferland, G. J., et al. The 2013 Release of Cloudy. Rev. Mexicana Astron. Astrofis. 49, 137-163 (2013).

[38] Schönberner, D., Jacob, R., Sandin, C. and Steffen, M., The evolution of planetary nebulae. VII. Modelling planetary nebulae of distant stellar systems. A\&SA 523, A86 (2010).

[39] Frew, D. J. and Parker, Q. A., Planetary Nebulae: Observational Properties, Mimics and Diagnostics PASA 27, 129-148 (2010) 


\title{
Acknowledgements
}

AAZ and KG acknowledge the financial support by The University of Manchester and by Nicolaus Copernicus University. AAZ is supported by the UK Science and Technology Facility Council (STFC) under grant ST/P000649/1. M3B is partially suported by ANPCyT and CONICET through grants PICT-2 014-2708 and PIP 112-200801-00940 and also by a Return Fellowship from the Alexander von Humboldt Foundation.

\section{Author informations}

\section{Affiliations}

K. Gesicki

Centre for Astronomy, Faculty of Physics, Astronomy and Informatics, Nicolaus Copernicus University, Grudziadzka 5, PL-87-100 Torun, Poland;

e-mail: kmgesicki@umk.pl

\author{
A. A. Zijlstra \\ Jodrell Bank Centre for Astrophysics, School of Physics \& Astronomy, \\ University of Manchester, Oxford Road, Manchester M13 9PL, UK; \\ Kong, Pok Fu Lam Road, Hong Kong; \\ e-mail: a.zijlstra@manchester.ac.uk \\ M. M. Miller Bertolami \\ Instituto de Astrofísica de La Plata, UNLP-CONICET, \\ Paseo del Bosque s/n, 1900 La Plata, Argentina; \\ e-mail: mmiller@fcaglp.unlp.edu.ar
}

Department of Physics \& Laboratory for Space Research, University of Hong

\section{Contributions}

AAZ and KG developed the concept. MMMB provided the post-AGB evolutionary sequences obtained with LPCODE and computed the supplementary data. KG adopted the Torun codes for the present work, performed the photoionization calculations, and synthesized the PNLF. All authors participated in discussions of the results, their presentations in figures and descriptions in manuscript and in pinpointing the conclusions.

\section{Competing interests}

The authors declare no competing financial interests.

\section{Corresponding author}

K.Gesicki 